\title{
Entanglement access control for the quantum Internet
}

\author{
Laszlo Gyongyosi ${ }^{1,2,3} \cdot$ Sandor Imre ${ }^{2}$
}

Received: 9 February 2018 / Accepted: 21 February 2019 / Published online: 28 February 2019

(c) The Author(s) 2019

\begin{abstract}
Quantum entanglement is a crucial element of establishing the entangled network structure of the quantum Internet. Here we define a method to achieve controlled entanglement access in the quantum Internet. The proposed model defines different levels of entanglement accessibility for the users of the quantum network. The path cost is determined by an integrated criterion on the entanglement fidelities between the quantum nodes and the probabilities of entangled connections of an entangled path. We reveal the connection between the number of available entangled paths and the accessible fidelity of entanglement and reliability in the end nodes. The scheme provides an efficient model for entanglement access control in the experimental quantum Internet.
\end{abstract}

Keywords Quantum Internet · Quantum repeater · Quantum entanglement · Quantum Shannon theory

\section{Introduction}

In the quantum Internet, the quantum nodes share quantum entanglement among one other, which provides an entangled ground-base network structure for the various quantum networking protocols [1-20]. In a quantum Internet scenario, the aim of the quantum repeater elements is to extend the range of entanglement through several steps [20-33]. The available entanglement at the end points has several critical parameters, most importantly the fidelity of the established entanglement (fidelity of entanglement

$凶$ Laszlo Gyongyosi

l.gyongyosi@soton.ac.uk

1 School of Electronics and Computer Science, University of Southampton, Southampton SO17 $1 \mathrm{BJ}, \mathrm{UK}$

2 Department of Networked Systems and Services, Budapest University of Technology and Economics, Budapest 1117, Hungary

3 MTA-BME Information Systems Research Group, Hungarian Academy of Sciences, Budapest 1051, Hungary 
$[34,35])$ and the probability of the existence of a given entangled connection $[1,8]$. In an experimental setting, these critical parameters are time varying since the noise of local quantum memories that store the shared entanglement in the quantum nodes evolves over time, and the probability of entangled connections (shared entanglement between a node pair) also changes dynamically [1,5,6,36-46].

In the quantum Internet, several entangled paths (paths formulated by several entangled connections) could exist between a given source-target quantum node pair $[1,5,6,45,47-63]$. This fact allows us to introduce a method that utilizes this multipath property to change these critical parameters via the number of entangled paths associated with a given end-to-end node pair: the available fidelity of entanglement and the probability of an entangled connection. The model utilizes the reliability (probability) of the entangled connections and the entanglement fidelity coefficient as primary metrics. The decomposition is motivated by the fact that a maximization of the entanglement throughput (number of transmitted Bell states per a given time unit at a particular fidelity) parameter requires also the maximization of the connection probability and the entanglement fidelity.

In this work, we define a method for entanglement access control in entangled quantum networks. The entanglement differentiation is achieved via a controlled variability of entanglement fidelity and entangled connection probability between source and target quantum nodes in a quantum repeater network. The proposed approach allows us to define different priority levels of entanglement access for the legal users of the quantum network with respect to the number of available paths. The number of available paths injects an additional degree of freedom to the quantum network, allowing for the selection of the entanglement fidelity and connection reliability for the end nodes. In a straightforward application of our method, the high-priority demands are associated with high fidelity and high connection probability in the end nodes of the user, while the lower-priority users get lower fidelity and lower connection probability in their end nodes. To achieve the differentiation, we define the appropriate cost and path cost functions and the criteria regarding the entanglement fidelity and connection probability for the quantum nodes and entangled connections of the entangled path. The entanglement differentiation utilizes a different number of paths between the source and target nodes allowing a distinction to be made between single-path and multipath scenarios. In a single-path setting, only one entangled path exists between source and target nodes, and therefore, the fidelity of entanglement and the probability of existence of the entangled connections between the end nodes are determined only by the nodes of the given entangled path. In a multipath scenario, more than one path exists from a source to a target.

In our model, a given criterion regarding the entanglement fidelity of the local nodes has to be satisfied for all node pairs on the path referred to as integrated connection probability and fidelity criterion for all entangled connections of the entangled path. The integrated criterion allows us to reach a given entanglement fidelity and a given connection probability between the end nodes of the quantum network.

Our solution utilizes time-varying parameters since the cost functions deal with the evolution of the entanglement fidelity parameter and the connection probabilities, which evolve over time. We define the entanglement access control algorithm for 
an arbitrary topology quantum repeater network. We also reveal the computational complexity of the method.

The novel contributions of our manuscript are as follows:

1. We define a method to achieve controlled entanglement accessibility in the quantum Internet.

2. The algorithm defines entangled paths between the source and target nodes in function of a particular path cost function.

3. The path cost is determined by an integrated criterion on the entanglement fidelity and the probability of entangled connection.

4. The proposed scheme has moderate complexity, providing an efficient entanglement accessibility differentiation, allowing for the construction of different priority levels of entanglement accessibility for users.

5. The results can be straightforwardly applied in the entangled quantum networks of the quantum Internet.

This paper is organized as follows. In Sect. 2, the basic components of the model are summarized. In Sect. 3, the entanglement accessibility methods are discussed. Section 4 proposes the integrated criterion related to entanglement fidelity. Section 5 defines the entanglement access control algorithm. Finally, Sect. 6 concludes the results.

\section{System model}

\subsection{Entangled network}

The quantum Internet setting is modeled as follows [8]. Let $V$ refer to the nodes of an entangled quantum network $N$, with a transmitter quantum node $A \in V$, a receiver quantum node $B \in V$, and quantum repeater nodes $R_{i} \in V, i=1, \ldots, q$. Let $E=\left\{E_{j}\right\}, j=1, \ldots, m$, refer to a set of edges between the nodes of $V$, where each $E_{j}$ identifies an $\mathrm{L}_{l}$-level entangled connection, $l=1, \ldots, r$, between quantum nodes $x_{j}$ and $y_{j}$ of edge $E_{j}$, respectively. The entanglement levels of the entangled connections in the entangled quantum network structure are defined as follows.

\subsubsection{Entanglement levels in the quantum Internet}

In a quantum Internet setting, an $N=(V, E)$ entangled quantum network consists of single-hop and multihop entangled connections, such that the single-hop entangled nodes ${ }^{1}$ are directly connected through an $\mathrm{L}_{1}$-level entanglement, while the multihop entangled nodes communicate through $\mathrm{L}_{l}$-level entanglement. Focusing on the doubling architecture $[1,5,6]$ in the entanglement distribution procedure, the number of spanned nodes is doubled in each level of entanglement swapping (entanglement swapping is applied in an intermediate node to create a longer distance entanglement

\footnotetext{
1 The $l$-level entangled nodes $x, y$ refer to quantum nodes $x$ and $y$ connected by an entangled connection $\mathrm{L}_{l}$.
} 
[1]). Therefore, the $d(x, y)_{\mathrm{L}_{l}}$ hop distance in $N$ for the $\mathrm{L}_{l}$-level entangled connection between $x, y \in V$ is denoted by [8]

$$
d(x, y)_{\mathrm{L}_{l}}=2^{l-1},
$$

with $d(x, y)_{\mathrm{L}_{l}}-1$ intermediate quantum nodes between $x$ and $y$. Therefore, $l=1$ refers to a direct entangled connection between two quantum nodes $x$ and $y$ without intermediate quantum repeaters, while $l>1$ identifies a multilevel entanglement.

\subsubsection{Entanglement fidelity}

Let

$$
\left|\beta_{00}\right\rangle=\frac{1}{\sqrt{2}}(|00\rangle+|11\rangle)
$$

be the target Bell state subject to be created at the end of the entanglement distribution procedure between a particular source node $A$ and receiver node $B$. The entanglement fidelity $F$ at an actually created noisy quantum system $\sigma$ between $A$ and $B$ is

$$
F(\sigma)=\left\langle\beta_{00}|\sigma| \beta_{00}\right\rangle,
$$

where $F$ is a value between 0 and $1, F=1$ for a perfect Bell state, and $F<1$ for an imperfect state. The $F$ entanglement fidelity represents the accuracy of our information about a quantum state $[1,5,6]$. The fidelity in (3) measures the amount of overlap between $\left|\beta_{00}\right\rangle$ target state (2) and the density matrix $\sigma$ that represents our system. In the entanglement distribution procedure, the usage of the $F$ entanglement fidelity metric rather than other correlation measure functions (concurrence, negativity, quantum discord, quantum coherent information, etc.) [4] is motivated by the fact that the fidelity of entanglement is an improvable parameter in a practical setting. The improvement of the fidelity is realizable by the so-called entanglement purification process [1]. The entanglement purification takes imperfect entangled states and outputs a higher-fidelity entangled system. Without loss of generality, in an experimental quantum Internet setting, an aim is to reach $F \geq 0.98$ over long distances $[1,5,6]$.

\subsubsection{Practical implementation}

An experimental quantum network refers to a set of source users (quantum nodes), destination users (quantum nodes), several intermediate quantum repeaters between them and to a set of physical node-to-node connections between the physical nodes (the physical attributes of the $l=1$ level connections identify the physical attributes of the physical links between the neighboring nodes). A quantum node is a quantum device with internal quantum memory $\mathcal{M}$ and with the capability of performing local operations (such as the internal processes connected to entanglement purification, entanglement swapping, error correction) [25,36-45,47-63]. In a practical setting, the node-to-node entanglement distribution can be implemented by an optical fiber network or via a wireless optical system (free-space channel [64] or a quantum-based 
satellite communication channel [27]). A physical link $\mathcal{N}$ is characterized by a particular link loss $\mathcal{L}(\mathcal{N})$. For a standard-quality optical fiber $\mathcal{N}$, the average link loss is $\mathcal{L}(\mathcal{N}) \approx 3.4 \mathrm{~dB}$, while the maximum of the tolerable link loss for an optical fiber system is $\mathcal{L}(\mathcal{N}) \approx 4.3 \mathrm{~dB}[1,6]$.

In an practical entangled quantum network, the $l>1$ level entangled connections refer to the case when the source and target quantum nodes are not directly connected by a physical link, but by an entangled connection that spans several quantum repeaters. An $l>1$ level entangled connection is formulated by several node-to-node interactions through the physical links in the physical layer.

\section{Entanglement access}

\subsection{Entanglement fidelity criterion}

First, we characterize the entanglement fidelity criterion for a given node pair. Using the criterion, we then derive the probability of the existence of single-path and multipath sets with $m$ end-to-end connection-disjoint entangled paths $[65,66]$ between source and target nodes. The end-to-end connection-disjoint entangled paths share no any common entangled connection between a source node $A$ and a receiver node $B$.

A given entangled connection $\mathrm{L}_{l}$ is characterized by a particular fidelity $F^{*}$, whose quantity classifies the entangled connection, such that $F^{*} \geq F_{\text {crit }}$, where $F_{\text {crit }}$ is a critical lower bound on the fidelity of entanglement.

Let $E(x, y)$ refer to the entangled connection between a node pair $(x, y)$, and let $F_{\Delta}(x, y)$ be the difference of the fidelity of entanglement in quantum nodes $x$ and $y$, as

$$
F_{\Delta}(x, y)=\left|F_{x}-F_{y}\right|<\hat{F}_{\Delta},
$$

where $\hat{F}_{\Delta}$ is a maximal allowed fidelity distance, $F_{x} \geq F_{\text {crit }}$, and $F_{y} \geq F_{\text {crit }}$. Since the entangled connections are assumed to be time varying in the network $[65,66]$, the probability that $F_{\Delta}(x, y)<\hat{F}_{\Delta}$ holds at a given time $t$ for an entangled connection $E_{\mathrm{L}_{l}}(x, y)$ is as

$$
\operatorname{Pr}\left(F_{\Delta}(x, y)<\hat{F}_{\Delta}\right)=\int_{0}^{\hat{F}_{\Delta}} \delta(z) \mathrm{d} z
$$

where $\delta\left(F_{\Delta}(x, y)\right)$ is the probability density function of entanglement fidelity distance.

\subsubsection{Single-path entanglement accessibility}

Let $\mathcal{P}^{S}$ refer to a single path between $A_{\rho, U_{k}}$ and $B_{\rho, U_{k}}, k=1, \ldots, K$, where $\rho$ is a demand, $A_{\rho, U_{k}}$ and $B_{\rho, U_{k}}$ are the sender and destination nodes associated with the demand $\rho$ of user $U_{k}$, and $K$ is the number of users. The single entangled path 
setting means that entanglement can be distributed from $A_{\rho, U_{k}}$ to $B_{\rho, U_{k}}$ through only one given path in the network $N$. Let it be assumed that $\mathcal{P}^{S}$ consists of $g$ entangled connections; then the $\operatorname{Pr}\left(\mathcal{P}^{S}\right)$ probability that a given single path $\mathcal{P}^{S}$ exists between $A_{\rho, U_{k}}$ and $B_{\rho, U_{k}}$ with the fidelity criterion is expressed as

$$
\begin{aligned}
\operatorname{Pr}\left(\mathcal{P}^{S}\right) & =\prod_{E_{\mathrm{L}_{l}}(x, y) \in \mathcal{P} S} \operatorname{Pr}\left(F_{\Delta}(x, y)<\hat{F}_{\Delta}\right) \\
& =\left(\int_{0}^{\hat{F}_{\Delta}} \delta(z) \mathrm{d} z\right)^{g} .
\end{aligned}
$$

\subsubsection{Multipath entanglement accessibility}

Let $\mathcal{P}_{i}^{M}, i=1, \ldots, m$, refer to the $i$ th multipath between $A_{\rho, U_{k}}$ and $B_{\rho, U_{k}}$, which means that entanglement can be distributed from $A_{\rho, U_{k}}$ to $B_{\rho, U_{k}}$ through a set $\mathcal{P}_{M}$ of $m$ end-to-end connection-disjoint entangled paths as $\mathcal{P}_{M}=\left\{\mathcal{P}_{1}^{M}, \ldots, \mathcal{P}_{m}^{M}\right\}$ in the network $N$. The $\operatorname{Pr}\left(\mathcal{P}_{M}\right)$ probability [65] that $A_{\rho, U_{k}}$ and $B_{\rho, U_{k}}$ share a common entanglement with the fidelity criterion is as

$$
\operatorname{Pr}\left(\mathcal{P}_{M}\right)=1-\prod_{S=1}^{m}\left(1-\operatorname{Pr}\left(\mathcal{P}^{S}\right)\right)=1-\prod_{S=1}^{m}\left(1-\left(\int_{0}^{\hat{F}_{\Delta}} \delta(z) \mathrm{d} z\right)^{g_{S}}\right)
$$

where $S$ is a path index, and $g_{S}$ is the number of entangled connections associated with $\mathcal{P}^{S}$.

Based on the distribution of $F_{\Delta}(x, y)$ fidelity distances between the node pairs of the network, the formulas of (6) and (7) can be derived in a more exact form. ${ }^{2}$

\section{Integrated criterion on connection probability and fidelity}

The integrated criterion extends the results of Sect. 3 to include the criterion on the probability of the existence of a given entangled connection between a node pair of a path. Using the integrated criterion on the connection probability and entanglement fidelity, we derive the probability of existence of single-path and multipath sets with $m$ end-to-end connection-disjoint entangled paths [65] between source and target nodes.

In our model, the fidelity of shared entanglement evolves in time for a given node pair $(x, y)$. For each quantum node $i$ at a time $t$, let $\Psi_{i}(t)$ be defined as

$$
\Psi_{i}(t)=\left(\operatorname{Pr}\left(E_{\mathrm{L}_{l}}(i, j, t)\right), F_{i}(t)\right)^{T},
$$

\footnotetext{
2 Assuming an exponential distribution of $F_{\Delta}(x, y), \operatorname{Pr}\left(\mathcal{P}^{S}\right)=\int_{0}^{\hat{F}_{\Delta}} \lambda \mathrm{e}^{-\lambda z} \mathrm{~d} z=1-\mathrm{e}^{-\lambda \hat{F}_{\Delta}}$, where $\lambda$ is a distribution coefficient, while $\operatorname{Pr}\left(\mathcal{P}_{M}\right)=1-\prod_{S=1}^{m}\left(1-\left(1-\mathrm{e}^{-\lambda \hat{F}_{\Delta}}\right)^{g_{S}}\right)$.
} 
where $\operatorname{Pr}\left(E_{\mathrm{L}_{l}}(i, j, t)\right)$ is the probability of an $\mathrm{L}_{l}$-level entangled connection with a node $j$ determined in node $i$ at a time $t$, while $F_{i}(t)$ is the fidelity of entanglement determined in node $i$ at a time $t$.

For a node pair $(x, y)$, according to local quantities, the following distance can be defined:

$$
\Delta\left(\operatorname{Pr}\left(E_{\mathrm{L}_{l}}(t)\right)\right)=\left|\operatorname{Pr}\left(E_{\mathrm{L}_{l}}(x, y, t)\right)-\operatorname{Pr}\left(E_{\mathrm{L}_{l}}(y, x, t)\right)\right|,
$$

where $\operatorname{Pr}\left(E_{\mathrm{L}_{l}}(x, y, t)\right)$ and $\operatorname{Pr}\left(E_{\mathrm{L}_{l}}(y, x, t)\right)$ are the connection probability quantities determined in nodes $x$ and $y$, and the fidelity distance $F_{\Delta}(t)$ is described by

$$
F_{\Delta}(t)=\left|F_{x}(t)-F_{y}(t)\right|
$$

where $F_{x}(t)$ and $F_{y}(t)$ are the fidelity quantities determined in nodes $x$ and $y$.

A distance of $\Psi_{x}(t)$ and $\Psi_{y}(t)$ for a node pair $(x, y)$ at a particular time $t$ is expressed via $\gamma_{x, y}(t)$, as

$$
\begin{aligned}
\gamma_{x, y}(t) & =\left|\Psi_{x}(t)-\Psi_{y}(t)\right| \\
& =\left(\left(\operatorname{Pr}\left(E_{\mathrm{L}_{l}}(x, y, t)\right)-\operatorname{Pr}\left(E_{\mathrm{L}_{l}}(y, x, t)\right)\right)^{2}+\left(F_{x}(t)-F_{y}(t)\right)^{2}\right)^{\frac{1}{2}}
\end{aligned}
$$

Since the connection probability and the entanglement fidelity parameters evolve over time, after $\Delta t$ from an initial time $t_{0}$, the quantity $\Psi_{x}\left(t_{0}+\Delta t\right)$ of a given node $x$ evolves as

$$
\Psi_{x}\left(t_{0}+\Delta t\right)=\Psi_{x}\left(t_{0}\right)+\chi_{x}\left(t_{0}, \Delta t\right)
$$

where $\chi_{x}\left(t_{0}, \Delta t\right)$ is expressed as

$$
\chi_{x}\left(t_{0}, \Delta t\right)=\left(\begin{array}{l}
\chi_{x}^{\operatorname{Pr}\left(E_{\mathrm{L}_{l}}(x, y)\right)}\left(t_{0}, \Delta t\right) \\
\chi_{x}^{F_{x}}\left(t_{0}, \Delta t\right)
\end{array}\right)=\left(\begin{array}{c}
\int_{t_{0}}^{t_{0}+\Delta t} \phi_{x}^{\operatorname{Pr}\left(E_{\mathrm{L}_{l}}(x, y)\right)}(q) \mathrm{d} q \\
\int_{t_{0}}^{t_{0}+\Delta t} \phi_{x}^{F_{x}}(q) \mathrm{d} q
\end{array}\right),
$$

where $\delta\left(\gamma_{x, y}\right)$ is the probability density function of distance function $\gamma_{x, y}$, and $\phi_{x}^{\operatorname{Pr}\left(E_{\mathrm{L}_{l}}(x, y)\right)}$ and $\phi_{x}^{F_{x}}$ are expressed as the connection probability and entanglement fidelity evolution functions of node $x$.

For a given node pair $(x, y)$, the particular upper bound $\gamma_{x, y}^{\max }$ on the maximal allowable distance between $\Psi_{x}\left(t_{0}+\Delta t\right)$ and $\Psi_{y}\left(t_{0}+\Delta t\right)$ at time $t_{0}+\Delta t$ leads to a limit, while $(x, y)$ can be referred to as entangled:

$$
\begin{aligned}
\gamma_{x, y}\left(t_{0}+\Delta t\right) & =\left|\Psi_{x}\left(t_{0}+\Delta t\right)-\Psi_{y}\left(t_{0}+\Delta t\right)\right| \\
& =\left|\Psi_{x}\left(t_{0}\right)+\chi_{x}\left(t_{0}, \Delta t\right)-\Psi_{y}\left(t_{0}\right)-\chi_{y}\left(t_{0}, \Delta t\right)\right| \leq \gamma_{x, y}^{\max } .
\end{aligned}
$$


If $\gamma_{x, y}\left(t_{0}+\Delta t\right)$ exceeds $\gamma_{x, y}^{\max }$, then the difference of the local entangled connection probabilities and entanglement fidelities is above a critical limit; therefore, the node pair $(x, y)$ is referred to as unentangled.

Using (11) and (13), (14) can be rewritten as

$$
\begin{aligned}
\gamma_{x, y}\left(t_{0}+\Delta t\right)= & \left(\left(\operatorname{Pr}\left(E_{\mathrm{L}_{l}}\left(x, y, t_{0}+\Delta t\right)\right)-\operatorname{Pr}\left(E_{\mathrm{L}_{l}}\left(y, x, t_{0}+\Delta t\right)\right)\right)^{2}\right. \\
& \left.+\left(F_{x}\left(t_{0}+\Delta t\right)-F_{y}\left(t_{0}+\Delta t\right)\right)^{2}\right)^{\frac{1}{2}}
\end{aligned}
$$

which leads to

$$
\begin{aligned}
& \gamma_{x, y}\left(t_{0}+\Delta t\right) \\
&=\left(\left(\operatorname{Pr}\left(E_{\mathrm{L}_{l}}\left(x, y, t_{0}\right)\right)+\int_{t_{0}}^{t_{0}+\Delta t} \phi_{x}^{\operatorname{Pr}\left(E_{\mathrm{L}_{l}}(x, y)\right)}(q) \mathrm{d} q\right.\right. \\
&\left.-\left(\operatorname{Pr}\left(E_{\mathrm{L}_{l}}\left(y, x, t_{0}\right)\right)+\int_{t_{0}}^{t_{0}+\Delta t} \phi_{y}^{\operatorname{Pr}\left(E_{\mathrm{L}_{l}}(y, x)\right)}(q) \mathrm{d} q\right)\right)^{2} \\
&\left.+\left(F_{x}\left(t_{0}\right)+\int_{t_{0}}^{t_{0}+\Delta t} \phi_{x}^{F_{x}}(q) \mathrm{d} q-\left(F_{y}\left(t_{0}\right)+\int_{t_{0}}^{t_{0}+\Delta t} \phi_{y}^{F_{y}}(q) \mathrm{d} q\right)\right)^{2}\right)^{\frac{1}{2}} .
\end{aligned}
$$

A representation of $F_{\Delta}\left(t_{0}\right)$ and $F_{\Delta}\left(t_{0}+\Delta t\right)$ for a node pair $(x, y)$ is depicted in Fig. 1. The $\operatorname{Pr}\left(E_{\mathrm{L}_{l}}(x, y)\right)$ connection probability is assumed to be different in the nodes at a particular time.

Fig. 1 Evolution of $F_{\Delta}(t)$ and $\operatorname{Pr}\left(E_{\mathrm{L}_{l}}(x, y)\right)$ for a node pair $(x, y)$ at $t=t_{0}$ and $t=t_{0}+\Delta t$. $F_{\Delta}\left(t_{0}\right)$ is $\left|F_{x}\left(t_{0}\right)-F_{y}\left(t_{0}\right)\right|$, where $F_{x}\left(t_{0}\right), F_{y}\left(t_{0}\right)$ are the fidelities of shared entanglement in the nodes, and $F_{\Delta}\left(t_{0}+\Delta t\right)$ yields the difference $\left|F_{x}\left(t_{0}+\Delta t\right)-F_{y}\left(t_{0}+\Delta t\right)\right|$

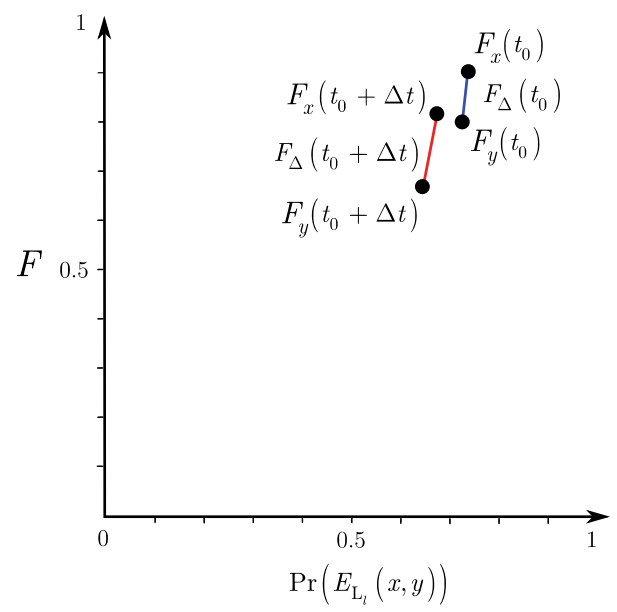




\subsection{Single-path entanglement accessibility}

After these derivations, the $\operatorname{Pr}\left(\mathcal{P}^{S}\left(t_{0}+\Delta t\right)\right)$ probability of a $\mathcal{P}^{S}$ single path in function of the connection probability and entanglement fidelity in the nodes of the path (e.g., connection probability criterion and fidelity criterion for all entangled connections of the path) is as follows.

Using $\gamma_{x, y}\left(t_{0}+\Delta t\right)$ in (16), the probability of the existence of a given single path $\mathcal{P}^{S}$ with $g_{S}$ entangled connections between $A_{\rho, U_{k}}$ and $B_{\rho, U_{k}}$ with a connection probability criterion and fidelity criterion for all entangled connections (see (6)) at time $t_{0}+\Delta t$ can therefore be rewritten as

$$
\begin{aligned}
& \operatorname{Pr}\left(\mathcal{P}^{S}\left(t_{0}+\Delta t\right)\right) \\
& =\prod_{E_{\mathrm{L}_{l}}(x, y) \in \mathcal{P}^{S}} \operatorname{Pr}\left(\gamma_{x, y}\left(t_{0}+\Delta t\right)<\gamma_{x, y}^{\max }\right) \\
& =\left(\int_{0}^{\gamma_{x, y}^{\max }} \delta(q) \mathrm{d} q\right)^{g_{S}},
\end{aligned}
$$

where

$$
\operatorname{Pr}\left(\gamma_{x, y}\left(t_{0}+\Delta t\right)<\gamma_{x, y}^{\max }\right)=\int_{0}^{\gamma_{x, y}^{\max }} \delta(q) \mathrm{d} q .
$$

\subsection{Multipath entanglement accessibility}

For the multipath scenario, (7) can be written via (16) as follows. For a given set of $m$ end-to-end connection-disjoint entangled paths expressed as $\mathcal{P}_{M}=\left\{\mathcal{P}_{1}^{M}, \ldots, \mathcal{P}_{m}^{M}\right\}$ between $A_{\rho, U_{k}}$ and $B_{\rho, U_{k}}$, the $\operatorname{Pr}\left(\mathcal{P}_{M}\left(t_{0}+\Delta t\right)\right)$ probability that $A_{\rho, U_{k}}$ and $B_{\rho, U_{k}}$ share a common entanglement with a connection probability criterion and fidelity criterion at time $t_{0}+\Delta t$ is expressed as

$$
\begin{aligned}
\operatorname{Pr}\left(\mathcal{P}_{M}\left(t_{0}+\Delta t\right)\right) & =1-\prod_{S=1}^{m}\left(1-\operatorname{Pr}\left(\mathcal{P}^{S}\left(t_{0}+\Delta t\right)\right)\right) \\
& =1-\prod_{S=1}^{m}\left(1-\left(\int_{0}^{\gamma_{x, y}^{\max }} \delta(q) \mathrm{d} q\right)^{g_{S}}\right) .
\end{aligned}
$$




\section{Control of entanglement access}

The entanglement access control algorithm establishes a number of connectiondisjoint entangled paths between a source node and a target node. Changing the number $m$ of connection-disjoint entangled paths allows us to modify both the probability of entanglement between the source and target nodes and the fidelity of available entanglement in the end nodes.

For the algorithm, a $c\left(E_{\mathrm{L}_{l}}(x, y)\right)$ cost function [65] of a given entangled connection $E_{\mathrm{L}_{l}}(x, y)$ is defined as

$$
\begin{aligned}
c\left(E_{\mathrm{L}_{l}}(x, y)\right)= & \left(\left(\chi_{x}^{\operatorname{Pr}\left(E_{\mathrm{L}_{l}}(x, y)\right)}\left(t_{0}, \Delta t\right)-\chi_{y}^{\operatorname{Pr}\left(E_{\mathrm{L}_{l}}(y, x)\right)}\left(t_{0}, \Delta t\right)\right)^{2}\right. \\
& \left.+\left(\chi_{x}^{F_{x}}\left(t_{0}, \Delta t\right)-\chi_{y}^{F_{y}}\left(t_{0}, \Delta t\right)\right)^{2}\right)^{\frac{1}{2}}
\end{aligned}
$$

where

$$
\begin{aligned}
& \chi_{x}^{\operatorname{Pr}\left(E_{\mathrm{L}_{l}}(x, y)\right)}\left(t_{0}, \Delta t\right)=\int_{t_{0}}^{t_{0}+\Delta t} \phi_{x}^{\operatorname{Pr}\left(E_{\mathrm{L}_{l}}(x, y)\right)}(q) \mathrm{d} q, \\
& \chi_{x}^{F_{x}}\left(t_{0}, \Delta t\right)=\int_{t_{0}}^{t_{0}+\Delta t} \phi_{x}^{F_{x}}(q) \mathrm{d} q,
\end{aligned}
$$

and

$$
\begin{array}{r}
\chi_{y}^{\operatorname{Pr}\left(E_{\mathrm{L}_{l}}(y, x)\right)}\left(t_{0}, \Delta t\right)=\int_{t_{0}}^{t_{0}+\Delta t} \phi_{y}^{\operatorname{Pr}\left(E_{\mathrm{L}_{l}}(y, x)\right)}(q) \mathrm{d} q, \\
\chi_{y}^{F_{y}}\left(t_{0}, \Delta t\right)=\int_{t_{0}}^{t_{0}+\Delta t} \phi_{y}^{F_{y}}(q) \mathrm{d} q .
\end{array}
$$

Let $N$ be the actual quantum repeater network with $|V|$ quantum nodes. A given $\mathrm{L}_{l}$-level entangled connection between a node pair $(x, y)$ is expressed as $E_{\mathrm{L}_{l}}(x, y)$.

Let $A_{\rho, U_{k}}$ and $B_{\rho, U_{k}}$ be the source and target quantum nodes of a demand $\rho$ associated with user $U_{k}$. Using (20) and a given entangled path $\mathcal{P}$ with a set of $q$ quantum repeaters $R_{i}, i=1, \ldots, q$, and a set $\mathcal{S}$ entangled connections, as

$$
\mathcal{S}=\left\{E_{\mathrm{L}_{l}}\left(A_{\rho, U_{k}}, R_{1}\right), \ldots, E_{\mathrm{L}_{l}}\left(R_{q}, B_{\rho, U_{k}}\right)\right\}
$$

the cost of path $\mathcal{P}$ is defined as

$$
c(\mathcal{P})=c\left(E_{\mathrm{L}_{l}}\left(A_{\rho, U_{k}}, R_{1}\right)\right)+\cdots+c\left(E_{\mathrm{L}_{l}}\left(R_{q}, B_{\rho, U_{k}}\right)\right) .
$$


The $\mathcal{D}_{\mathcal{A}}$ entanglement access control algorithm outputs a set of $\mathcal{P}_{M}=\left\{\mathcal{P}_{1}^{M}, \ldots, \mathcal{P}_{m}^{M}\right\}$, which contains the $m$ connection-disjoint entangled paths between $A_{\rho}, U_{k}$ and $B_{\rho, U_{k}}$.

In function of $m, \mathcal{U}_{C}$ priority classes can be defined for the users of the quantum Internet. A high-priority user gets a high value of $m$, while lower-priority users get lower values of $m$. The actual value of $m$ for a particular user class $\mathcal{U}_{C}$ can be determined in function of the current network conditions.

The steps are given in Algorithm 1.

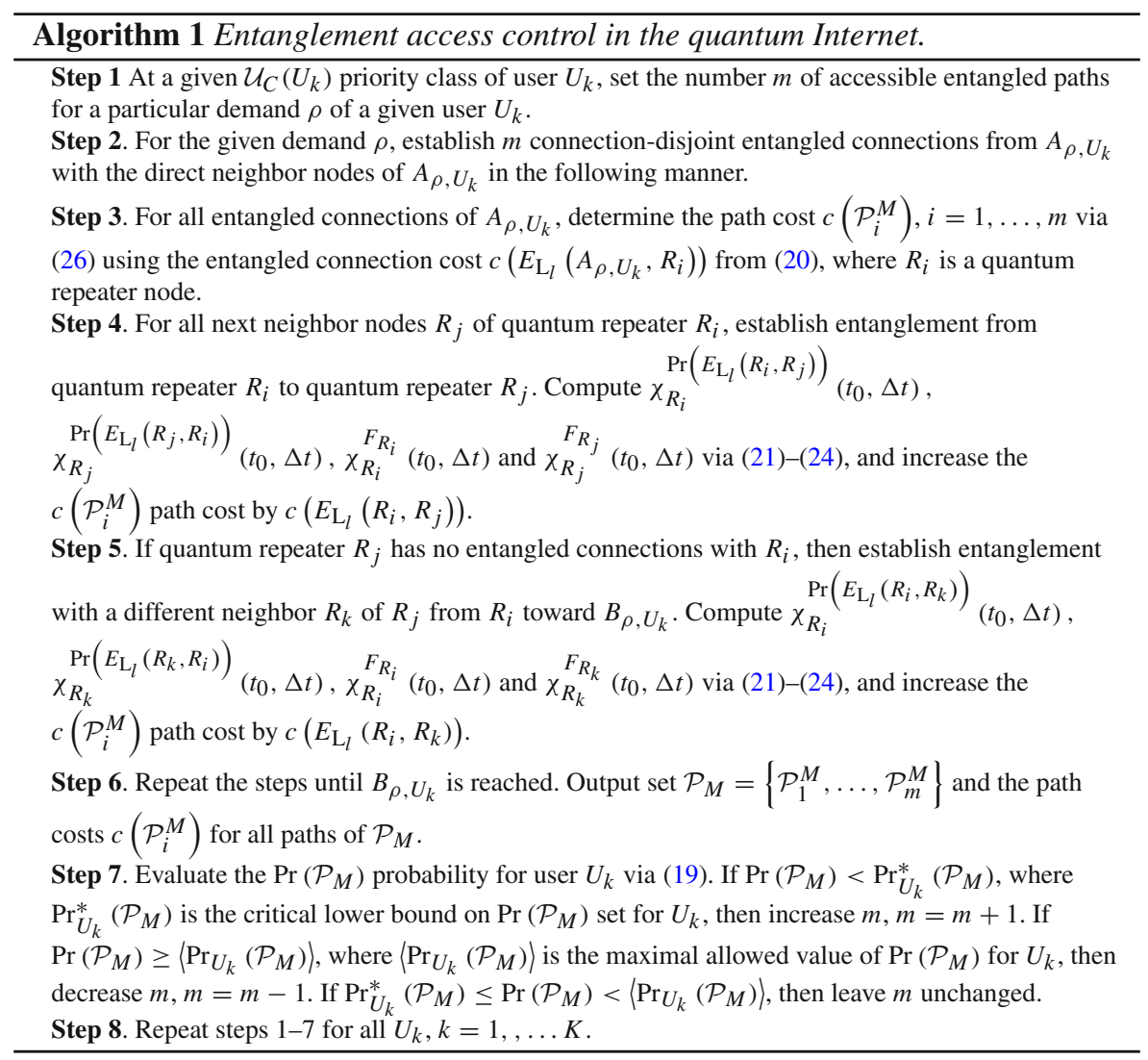

\subsection{Description}

A brief description of the $\mathcal{D}_{\mathcal{A}}$ entanglement access control algorithm is as follows.

Step 1 sets $m$ for a user $U_{k}$ by the $\mathcal{U}_{C}\left(U_{k}\right)$ priority class of the user. The $\mathcal{U}_{C}\left(U_{k}\right)$ determines the available value(s) of $m$ for $U_{k}$.

In Step 2, entanglement is established between the source node $A_{\rho, U_{k}}$ of the given demand of the user and the neighboring quantum repeaters. The relevant metrics quantities are also calculated in this step. 


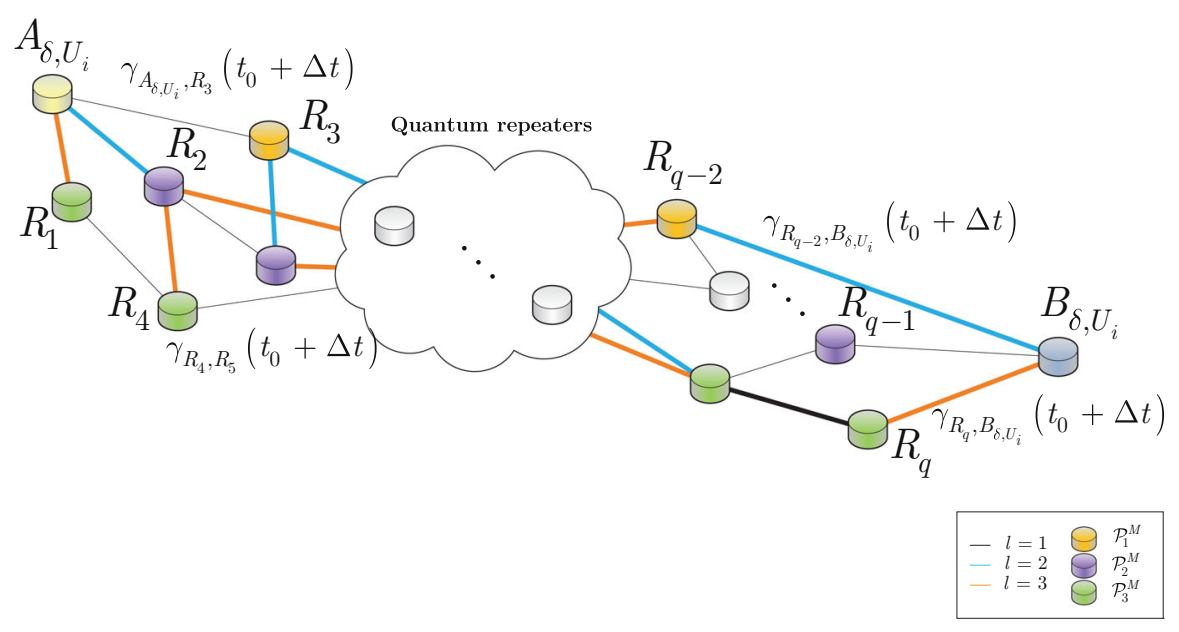

Fig. 2 A quantum Internet setting with $m=3$ connection-disjoint entangled paths $\mathcal{P}_{1}^{M}, \mathcal{P}_{2}^{M}$ and $\mathcal{P}_{3}^{M}$ between an $i$ th source quantum node $A_{\delta, U_{i}}$ and target quantum node $B_{\delta, U_{i}}$ with demand $\rho$, and $q$ intermediate $R_{i}$ quantum repeaters, $i=1, \ldots, q$. The entangled paths consist of $l=1$ level (direct) and multilevel, $l=2,3$ level entangled connections. The quantum nodes of $\mathcal{P}_{1}^{M}$ are depicted by orange, the nodes of $\mathcal{P}_{2}^{M}$ by purple, and the nodes of $\mathcal{P}_{3}^{M}$ by green. The $\gamma_{x, y}\left(t_{0}+\Delta t\right)$ coefficients are derived for all entangled connections of the paths

Using the derived quantities of Step 2, in Step 3, the cost paths are derived via (26) using the entangled connection cost formula of (20).

Steps 4-5 deal with the intermediate quantum repeater nodes associated with the given demand. These steps also ensure that entanglement is distributed through the cheapest path $c\left(\mathcal{P}^{\prime}\right)$ from a source node $A_{\rho, U_{k}}$ toward $B_{\rho, U_{k}}$, via a given intermediate repeater node $R_{i}$. It is ensured in our model that if the intermediate repeater node $R_{i}$ also shares entanglement with a quantum repeater $R_{j}$, then node $R_{j}$ will not establish entanglement with $B_{\rho, U_{k}}$, since $B_{\rho, U_{k}}$ can be reached via $R_{i}$, which is on the cheapest path $c\left(\mathcal{P}^{\prime}\right)$.

Step 6 outputs the set of $m$ end-to-end connection-disjoint entangled paths between $A_{\rho, U_{k}}$ and $B_{\rho, U_{k}}$ and the path costs for all paths.

Step 7 determines the $\operatorname{Pr}\left(\mathcal{P}_{M}\right)$ probability for user $U_{k}$ via (19) and updates the actual value of $m$ if needed.

Finally, Step 8 extends the steps for all users.

In Fig. 2 a multipath entanglement accessibility is depicted in a quantum Internet setting with heterogeneous entanglement levels. The network situation depicts connection-disjoint entangled paths that share no common entangled connection between a source node $A_{\delta, U_{i}}$ and a receiver node $B_{\delta, U_{i}}$. The entangled paths are characterized by the derived formulas.

\subsection{Computational complexity}

For a given quantum network $N$ with $|V|$ quantum nodes, the computational complexity of the $\mathcal{D}_{\mathcal{A}}$ entanglement access control algorithm for a given demand $\rho$ is at most 


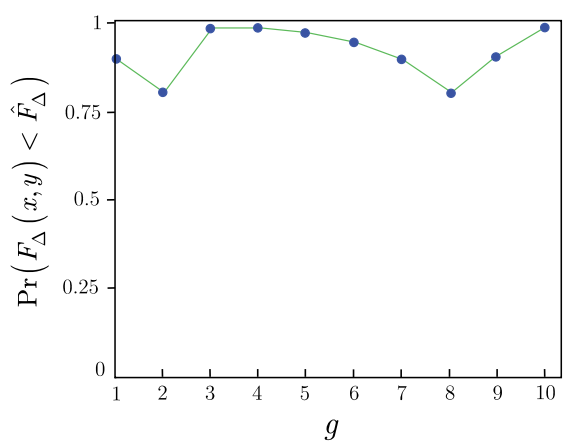

(a)

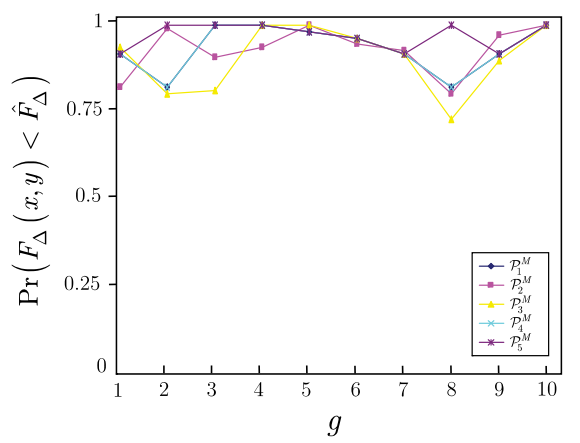

(b)

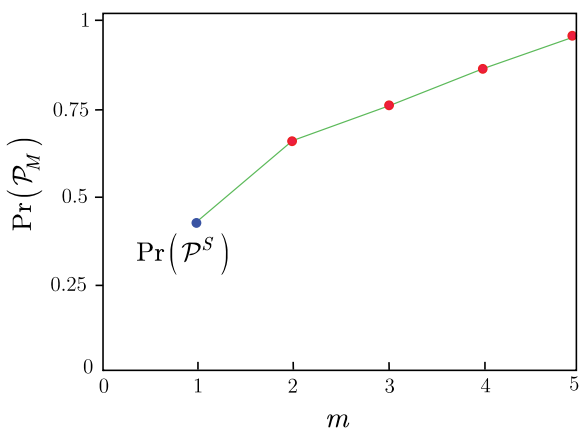

(c)

Fig. 3 The probability of shared entanglement between the source and the destination with the fidelity criterion at a single entangled path and at a multipath setting, $F_{\text {crit }}=0.98, \hat{F}_{\Delta}=0.02, g_{S}=g=10$. a A distribution of the $\left.\operatorname{Pr} F_{\Delta}(x, y)<\hat{F}_{\Delta}\right)$ probabilities for the $g$ entangled connections of a $\mathcal{P}^{S}$ single path. b A distribution of the $\operatorname{Pr}\left(F_{\Delta}(x, y)<\hat{F}_{\Delta}\right)$ probabilities for the $g_{S}=g$ entangled connections of a $\mathcal{P}_{M}=\left\{\mathcal{P}_{1}^{M}, \ldots, \mathcal{P}_{m}^{M}\right\}$ multipath setting, $m=5$. $\mathbf{c}$ The $\operatorname{Pr}\left(\mathcal{P}_{M}\right)$ probabilities in function of $m$. The $\operatorname{Pr}\left(\mathcal{P}_{S}\right)$ single-path probability is yielded at $m=1$

$\mathcal{O}(|V|)$, since the problem is analogous to the establishment of a path by message broadcasting [65].

\subsection{Numerical evidence}

We provide a numerical evidence on the distribution of the $\mathcal{P}^{S}$ and $\mathcal{P}_{M}$ path probabilities.

Let us set $F_{\text {crit }}=0.98$ for the lower bound on the fidelity of entanglement between all node pairs $x$ and $y, F_{x} \geq F_{\text {crit }}$, and $F_{y} \geq F_{\text {crit }}$. Then, the maximal allowed fidelity distance is set as $\hat{F}_{\Delta}=1-0.98=0.02$.

Then, let us assume that a $\mathcal{P}^{S}$ single path between $A_{\rho, U_{k}}$ and $B_{\rho, U_{k}}$ consists of $g$ entangled connections with different $l$ entanglement levels between the nodes of the path $\mathcal{P}^{S}$. For the $\mathcal{P}_{M}=\left\{\mathcal{P}_{1}^{M}, \ldots, \mathcal{P}_{m}^{M}\right\}$ multipath scenario, each entangled path 
consists of $g_{S}$ entangled connections with different $l$ entanglement levels between the nodes of each entangled path of $\mathcal{P}_{M}$.

For simplicity let us assume that the number of entangled connections is set as $g_{S}=g=10$ for all entangled paths, and the distribution of the $\operatorname{Pr}\left(F_{\Delta}(x, y)<\hat{F}_{\Delta}\right)$ probabilities for the entangled connections of a $\mathcal{P}^{S}$ single path at $\hat{F}_{\Delta}=0.02$ is as depicted in Fig. 3a. The distribution of the $\operatorname{Pr}\left(F_{\Delta}(x, y)<\hat{F}_{\Delta}\right)$ probabilities for the entangled connections of a $\mathcal{P}_{M}$ multipath with $m=5$ at $\hat{F}_{\Delta}=0.02$ is distributed as depicted in Fig. 3b. The resulting $\operatorname{Pr}\left(\mathcal{P}^{S}\right)$ and $\operatorname{Pr}\left(\mathcal{P}_{M}\right)$ probabilities are depicted in Fig. 3c.

The numerical analysis revealed that $\operatorname{Pr}\left(\mathcal{P}_{S}\right) \approx 0.4171$ for a $\mathcal{P}_{S}$ single entangled path at the particular $\operatorname{Pr}\left(F_{\Delta}(x, y)<\hat{F}_{\Delta}\right)$ connection-level values of the path (given in Fig. 3a). The $\mathcal{P}_{M}$ multipath setting at connection-level values of Fig. 3b, at $m=4$, doubles the success probability of the single-path setting with $\operatorname{Pr}\left(\mathcal{P}_{M}\right) \approx 0.8549$, while at $m=5$, the resulting probability is $\operatorname{Pr}\left(\mathcal{P}_{M}\right) \approx 0.9476$.

\section{Conclusions}

In this work, we defined a method to achieve entanglement access control in the quantum Internet. The algorithm utilizes different paths between the source and target nodes in function of a particular path cost function. The path cost function uses the local entanglement fidelities of the nodes and the probability of the existence of the entangled connections. Increasing the number of available paths leads to a multipath setting, which allows the parties to establish high fidelity entanglement with reliable entangled connections between the end nodes. The proposed scheme has moderate complexity, and it is particularly convenient for the entangled quantum network structure of the quantum Internet.

Acknowledgements Open access funding provided by Budapest University of Technology and Economics (BME). This work was partially supported by the National Research Development and Innovation Office of Hungary (Project No. 2017-1.2.1-NKP-2017-00001), by the Hungarian Scientific Research Fund - OTKA K-112125, and in part by the BME Artificial Intelligence FIKP Grant of EMMI (BME FIKP-MI/SC).

Author contributions LGY designed the protocol and wrote the manuscript. LGY and SI analyzed the results. All authors reviewed the manuscript.

\section{Compliance with ethical standards}

Conflict of interest The authors declare that they have no conflict of interest.

Open Access This article is distributed under the terms of the Creative Commons Attribution 4.0 International License (http://creativecommons.org/licenses/by/4.0/), which permits unrestricted use, distribution, and reproduction in any medium, provided you give appropriate credit to the original author(s) and the source, provide a link to the Creative Commons license, and indicate if changes were made. 


\section{References}

1. Van Meter, R.: Quantum Networking. Wiley (2014). ISBN: 1118648927,9781118648926

2. Lloyd, S., Shapiro, J.H., Wong, F.N.C., Kumar, P., Shahriar, S.M., Yuen, H.P.: Infrastructure for the quantum internet. ACM SIGCOMM Comput. Commun. Rev. 34, 9-20 (2004)

3. Kimble, H.J.: The quantum internet. Nature 453, 1023-1030 (2008)

4. Gyongyosi, L., Imre, S., Nguyen, H.V.: A survey on quantum channel capacities. IEEE Commun. Surv. Tutor. (2018). https://doi.org/10.1109/COMST.2017.2786748

5. Van Meter, R., Ladd, T.D., Munro, W.J., Nemoto, K.: System design for a long-line quantum repeater. IEEE/ACM Trans. Netw. 17(3), 1002-1013 (2009)

6. Van Meter, R., Satoh, T., Ladd, T.D., Munro, W.J., Nemoto, K.: Path selection for quantum repeater networks. Netw. Sci. 3(1-4), 82-95 (2013)

7. Van Meter, R., Devitt, S.J.: Local and distributed quantum computation. IEEE Comput. 49(9), 31-42 (2016)

8. Gyongyosi, L., Imre, S.: Decentralized base-graph routing for the quantum internet. Phys. Rev. A (2018). https://doi.org/10.1103/PhysRevA.98.022310

9. Gyongyosi, L., Imre, S.: Dynamic topology resilience for quantum networks. In: Proceedings of the SPIE 10547, Advances in Photonics of Quantum Computing, Memory, and Communication XI, 105470Z (2018). https://doi.org/10.1117/12.2288707

10. Gyongyosi, L., Imre, S.: Topology adaption for the quantum internet. Quantum Inf. Process. 17, 295 (2018). https://doi.org/10.1007/s11128-018-2064-X

11. Gyongyosi, L., Imre, S.: Adaptive routing for quantum memory failures in the quantum internet. Quantum Inf. Process. (2018). https://doi.org/10.1007/s11128-018-2153-x

12. Pirandola, S., Laurenza, R., Ottaviani, C., Banchi, L.: Fundamental limits of repeaterless quantum communications. Nat. Commun. 15043 (2017). https://doi.org/10.1038/ncomms15043

13. Pirandola, S., Braunstein, S.L., Laurenza, R., Ottaviani, C., Cope, T.P.W., Spedalieri, G., Banchi, L.: Theory of channel simulation and bounds for private communication. Quantum Sci. Technol. 3, 035009 (2018)

14. Pirandola, S. Capacities of repeater-assisted quantum communications. arXiv:1601.00966 (2016)

15. Laurenza, R., Pirandola, S.: General bounds for sender-receiver capacities in multipoint quantum communications. Phys. Rev. A 96, 032318 (2017)

16. Gyongyosi, L., Imre, S.: Multilayer optimization for the quantum internet. Sci. Rep. (2018). https:// doi.org/10.1038/s41598-018-30957-x

17. Gyongyosi, L., Imre, S.: Entanglement availability differentiation service for the quantum internet. Sci. Rep. (2018). https://doi.org/10.1038/s41598-018-28801-3. https://www.nature.com/articles/s41598018-28801-3

18. Gyongyosi, L., Imre, S.: Entanglement-gradient routing for quantum networks. Sci. Rep. (2017). https:// doi.org/10.1038/s41598-017-14394-w. https://www.nature.com/articles/s41598-017-14394-w

19. Imre, S., Gyongyosi, L.: Advanced Quantum Communications: An Engineering Approach. WileyIEEE Press, Hoboken (2013)

20. Caleffi, M.: End-to-end entanglement rate: toward a quantum route metric. In: 2017 IEEE Globecom (2018). https://doi.org/10.1109/GLOCOMW.2017.8269080

21. Caleffi, M.: Optimal routing for quantum networks. IEEE Access 5, 22299 (2017). https://doi.org/10. 1109/ACCESS.2017.2763325

22. Caleffi, M., Cacciapuoti, A.S., Bianchi, G.: Quantum internet: from communication to distributed computing. arXiv:1805.04360 (2018)

23. Castelvecchi, D.: The quantum internet has arrived. Nature 554, 289-292 (2018)

24. Cacciapuoti, A.S., Caleffi, M., Tafuri, F., Cataliotti, F.S., Gherardini, S., Bianchi, G.: Quantum internet: networking challenges in distributed quantum computing. arXiv:1810.08421 (2018)

25. Kok, P., Munro, W.J., Nemoto, K., Ralph, T.C., Dowling, J.P., Milburn, G.J.: Linear optical quantum computing with photonic qubits. Rev. Mod. Phys. 79, 135-174 (2007)

26. Petz, D.: Quantum Information Theory and Quantum Statistics. Springer, Heidelberg (2008)

27. Bacsardi, L.: On the way to quantum-based satellite communication. IEEE Commun. Mag. 51(08), 50-55 (2013)

28. Biamonte, J., et al.: Quantum machine learning. Nature 549, 195-202 (2017)

29. Lloyd, S., Mohseni, M., Rebentrost, P.: Quantum algorithms for supervised and unsupervised machine learning. arXiv:1307.0411 (2013) 
30. Lloyd, S., Mohseni, M., Rebentrost, P.: Quantum principal component analysis. Nat. Phys. 10, 631 (2014)

31. Lloyd, S.: Capacity of the noisy quantum channel. Phys. Rev. A 55, 1613-1622 (1997)

32. Lloyd, S.: The universe as quantum computer. In: Zenil, H. (ed.) A Computable Universe: Understanding and Exploring Nature as Computation. World Scientific, Singapore. arXiv:1312.4455v1 (2013)

33. Shor, P.W.: Scheme for reducing decoherence in quantum computer memory. Phys. Rev. A 52, R2493R2496 (1995)

34. Nielsen, M.A.: The entanglement fidelity and quantum error correction. arXiv:quant-ph/9606012 (1996)

35. Schumacher, B.: Sending quantum entanglement through noisy channels. Phys Rev A. 54(4), 26142628 (1996)

36. Chou, C., Laurat, J., Deng, H., Choi, K.S., de Riedmatten, H., Felinto, D., Kimble, H.J.: Functional quantum nodes for entanglement distribution over scalable quantum networks. Science 316(5829), 1316-1320 (2007)

37. Muralidharan, S., Kim, J., Lutkenhaus, N., Lukin, M.D., Jiang, L.: Ultrafast and fault-tolerant quantum communication across long distances. Phys. Rev. Lett. 112, 250501 (2014)

38. Yuan, Z., Chen, Y., Zhao, B., Chen, S., Schmiedmayer, J., Pan, J.W.: Experimental demonstration of a BDCZ quantum repeater node Nature 454, 1098-1101 (2008)

39. Kobayashi, H., Le Gall, F., Nishimura, H., Rotteler, M.: General scheme for perfect quantum network coding with free classical communication. In: Lecture Notes in Computer Science (Automata, Languages and Programming SE-52) vol. 5555, pp. 622-633. Springer (2009)

40. Hayashi, M.: Prior entanglement between senders enables perfect quantum network coding with modification. Phys. Rev. A 76, 040301(R) (2007)

41. Hayashi, M., Iwama, K., Nishimura, H., Raymond, R., Yamashita, S.: Quantum network coding. In: Thomas, W., Weil, P. (eds.) Lecture Notes in Computer Science (STACS 2007 SE52), vol. 4393. Springer, Berlin (2007)

42. Chen, L., Hayashi, M.: Multicopy and stochastic transformation of multipartite pure states. Phys. Rev. A 83(2), 022331 (2011)

43. Schoute, E., Mancinska, L., Islam, T., Kerenidis, I., Wehner, S.: Shortcuts to quantum network routing. arXiv:1610.05238 (2016)

44. Lloyd, S., Weedbrook, C.: Quantum generative adversarial learning. Phys. Rev. Lett. 121. arXiv:1804.09139 (2018)

45. Gisin, N., Thew, R.: Quantum communication. Nat. Photon. 1, 165-171 (2007)

46. Shor, P.W.: Fault-tolerant quantum computation. In: 37th Symposium on Foundations of Computing. IEEE Computer Society Press, pp. 56-65 (1996)

47. Xiao, Y.F., Gong, Q.: Optical microcavity: from fundamental physics to functional photonics devices. Sci. Bull. 61, 185-186 (2016)

48. Zhang, W., et al.: Quantum secure direct communication with quantum memory. Phys. Rev. Lett. 118, 220501 (2017)

49. Gyongyosi, L., Imre, S.: A survey on quantum computing technology. Comput. Sci. Rev. (2018). https://doi.org/10.1016/j.cosrev.2018.11.002. ISSN: 1574-0137

50. Enk, S.J., Cirac, J.I., Zoller, P.: Photonic channels for quantum communication. Science 279, 205-208 (1998)

51. Briegel, H.J., Dur, W., Cirac, J.I., Zoller, P.: Quantum repeaters: the role of imperfect local operations in quantum communication. Phys. Rev. Lett. 81, 5932-5935 (1998)

52. Dur, W., Briegel, H.J., Cirac, J.I., Zoller, P.: Quantum repeaters based on entanglement purification. Phys. Rev. A 59, 169-181 (1999)

53. Duan, L.M., Lukin, M.D., Cirac, J.I., Zoller, P.: Long-distance quantum communication with atomic ensembles and linear optics. Nature 414, 413-418 (2001)

54. Van Loock, P., Ladd, T.D., Sanaka, K., Yamaguchi, F., Nemoto, K., Munro, W.J., Yamamoto, Y.: Hybrid quantum repeater using bright coherent light. Phys. Rev. Lett. 96, 240501 (2006)

55. Zhao, B., Chen, Z.B., Chen, Y.A., Schmiedmayer, J., Pan, J.W.: Robust creation of entanglement between remote memory qubits. Phys. Rev. Lett. 98, 240502 (2007)

56. Goebel, A.M., Wagenknecht, G., Zhang, Q., Chen, Y., Chen, K., Schmiedmayer, J., Pan, J.W.: Multistage entanglement swapping. Phys. Rev. Lett. 101, 080403 (2008)

57. Simon, C., de Riedmatten, H., Afzelius, M., Sangouard, N., Zbinden, H., Gisin, N.: Quantum repeaters with photon pair sources and multimode memories. Phys. Rev. Lett. 98, 190503 (2007) 
58. Tittel, W., Afzelius, M., Chaneliere, T., Cone, R.L., Kroll, S., Moiseev, S.A., Sellars, M.: Photon-echo quantum memory in solid state systems. Laser Photon. Rev. 4, 244-267 (2009)

59. Sangouard, N., Dubessy, R., Simon, C.: Quantum repeaters based on single trapped ions. Phys. Rev. A 79, 042340 (2009)

60. Dur, W., Briegel, H.J.: Entanglement purification and quantum error correction. Rep. Prog. Phys 70, 1381-1424 (2007)

61. Sheng, Y.B., Zhou, L.: Distributed secure quantum machine learning. Sci. Bull. 62, 1019-1025 (2017)

62. Leung, D., Oppenheim, J., Winter, A.: Quantum network communication: the butterfly and beyond. IEEE Trans. Inf. Theory 56, 3478-90 (2010)

63. Kobayashi, H., Le Gall, F., Nishimura, H., Rotteler, M.: Perfect quantum network communication protocol based on classical network coding. In: Proceedings of 2010 IEEE International Symposium on Information Theory (ISIT), pp. 2686-2690 (2010)

64. Fedrizzi, A., Ursin, R., Herbst, T., Nespoli, M., Prevedel, R., Scheidl, T., Tiefenbacher, F., Jennewein, T., Zeilinger, A.: High-fidelity transmission of entanglement over a high-loss free-space channel. Nat. Phys. 5(6), 389-392 (2009)

65. Rak, J.: Resilient Routing in Communication Networks. Springer, Berlin (2015)

66. Rak, J.: k-Penalty: a novel approach to find k-disjoint paths with differentiated path costs. IEEE Commun. Lett. 14(4), 354-356 (2010)

Publisher's Note Springer Nature remains neutral with regard to jurisdictional claims in published maps and institutional affiliations. 